\title{
NOTAS
}

\section{Cerro Conde: estudio preliminar de un sitio arqueológico situado en la margen derecha del valle medio del río Chillón}

\section{Cerro Conde: preliminary study of an archaeological site located on the right bank of the middle valley of Chillón river}

\author{
Anthony Ulises Villalta Tello \\ https://orcid.org/0000-0003-2689-1051 \\ Universidad Nacional Mayor de San Marcos \\ anthony.vt@hotmail.com
}

\section{INTRODUCCIÓN}

El presente estudio es producto de las investigaciones que realizamos durante el curso de Prácticas pre-profesionales en Arqueología III, dictado por la Universidad Nacional Mayor de San Marcos, abarca los estudios realizados a nivel superficial del sitio arqueológico Cerro Conde que se ubica en el Centro Poblado Huarangal (ExHacienda Huarangal), distrito de Carabayllo, Lima. El pionero en hacer estudios de los asentamientos arqueológicos del valle del río Chillón fue el padre Villar Córdova (1935), posteriormente investigadores como Dillehay (1976), Rostorowski (1977), Silva (1996), Farfán (2000), y Aranguren (2002), han puesto los bases para el futuro estudio de esta zona. Luego de la revisión bibliográfica en general del valle del Chillón, decidí investigar los asentamientos arqueológicos que están en el límite del valle bajo y medio del río Chillón, específicamente los asentamientos que se posicionaron en las laderas y las cumbres de los cerros que están próximos al río Chillón. Luego 
de delimitar el área de estudio, plantear la problemática e identificar el objeto de estudio, decidí investigar el sitio arqueológico Cerro Conde, ya que es un sitio que cumple con las características mencionadas líneas arriba.

\section{UBICACIÓN Y EXTENSIÓN}

La cuenca del río Chillón se encuentra ubicado entre la provincia de Canta (cuenca alta y media) y la provincia de Lima (parte de la cuenca media y baja), departamento de Lima. El río Chillón es integrante de la cuenca hidrográfica del Océano Pacífico, este limita al sur con la cuenca del río Rímac (provincia de Lima), al norte con la cuenca del río Chancay (provincia de Huaral), al este con la cuenca del río Mantaro (Junín) y al oeste con el Océano Pacífico. La cuenca alta del río Chillón desciende desde su formación a una altura de $4800 \mathrm{~m} \mathrm{~s}$. n. m. hasta los 2500-2000 m s. n. m., la cuenca media desciende desde los $2000 \mathrm{~m} \mathrm{~s}$. n. m. hasta 500-400 m s. n. m. y la cuenca baja desde los 400 m s. n. m. hasta 0 m s. n. m., desembocando en el Océano Pacífico.

\section{EL RÍO CHILLÓN: CARACTERÍSTICAS}

El río Chillón tiene su origen en las lagunas de Pucracocha, Aguascocha y Chuchón (Vera, 2002) que discurre de este a oeste, que se encuentran al pie de la Cordillera de la Viuda, en el límite de la provincia de Canta hasta desembocar en el Océano Pacífico, alimentándose con las precipitaciones, con los deshielos de la cordillera de la Viuda y los afluentes que discurren en ambas márgenes del valle. La cuenca del río Chillón se encuentra ubicada entre las coordenadas geográficas $76^{\circ} 28^{\prime}$ y $77^{\circ} 10^{\prime}$ de longitud Oeste y $11^{\circ} 20^{\prime}$ y $12^{\circ} 15^{\prime}$ de latitud sur y tiene un recorrido de $126 \mathrm{~km}$ hasta desembocar en el Océano Pacífico (INRENA, 2003). Políticamente el río Chillón recorre desde su naciente en la cumbre de la Cordillera de la Viuda en la provincia de Canta, pasando por los distritos de Arahuay, Canta, Huamantanga, Huaros, Lachaqui, San Buenaventura y Santa Rosa de Quives; en la provincia de Lima por los distritos de Carabayllo, Puente Piedra, Comas, Los olivos, San Martin de Porres y Ventanilla en donde desemboca (INRENA, 2003).

\section{SITUACIÓN PROBLEMÁTICA}

El Complejo Arqueológico de Cerro Conde se ubica a $100 \mathrm{~m}$ del Centro Poblado Huarangal, en la margen derecha del río Chillón. Políticamente se encuentra ubicado en el centro Poblado de Huarangal, distrito de Carabayllo y provincia de Lima. El complejo abarca una gran extensión sobre la ladera y cumbre del Cerro Conde. Según Jorge Silva (1996), el sitio presentaría ocupación durante el Periodo Intermedio Tardío (1100-1440 d.C), (van Dalen, 2012) asociándolo al complejo Huarangal. Se desconoce 
las características funcionales que se llevaron a cabo en este gran centro arquitectónico, durante el Periodo Intermedio Tardío.

Esta investigación comprende la descripción y el estudio de las evidencias arquitectónicas y la funcionalidad del sitio arqueológico Cerro Conde que se desarrolló durante el periodo intermedio tardío (S.XI-XV). Silva (1987), hace un estudio sobre el patrón de asentamientos de los diferentes asentamientos que se encuentran en la parte baja, media y alta del valle, catalogando así, que los asentamientos para el valle medio del río Chillón, están asentados en las terrazas aluviales, las laderas y en las cumbres de los cerros y que estos distintos asentamientos estarían cumpliendo diferentes funciones, en torno al manejo del espacio y sus recursos, como las zonas agrícolas y el agua.

En el caso del sitio arqueológico Cerro Conde, este se ubica en el valle medio, en la margen derecha del río Chillón, en donde está ubicado en la ladera y cumbre del cerro Conde. Este sitio ha sido construido sobre esta colina en donde se puede distinguir diferentes tipos de conjuntos arquitectónicos, como recintos circulares, recintos cuadrangulares e irregulares, terrazas, escalinatas, etc.; construidas con piedra canteada y que actualmente no se conservan en buen estado ya sean por factores antrópicos o naturales que han dañado sus estructuras. Las investigaciones realizadas por el Dr. Silva sobre los patrones de asentamientos de los sitios arqueológicos para el Intermedio Tardío, describe de manera general algunas terrazas y recintos del sitio arqueológico Cerro Conde, no ha habido un estudio general del sitio en donde se detalle el tipo de función o funciones y filiación cultural que se estarían dando para el Periodo Intermedio Tardío. De la revisión general de antecedentes de estudios que se han hecho para el sitio arqueológico Cerro Conde, en cuanto a las función o funciones y filiación cultural que se desarrollaron para este sitio durante el Periodo Intermedio Tardío, son escasos. De las cuales nos llevan a formular problemas tales como: ¿Cuáles fueron la función o funciones que se desempeñaron en el sitio arqueológico Cerro Conde?, en cuanto a la filiación crono-cultural ¿el sitio Arqueológico Cerro Conde sería parte integrante de la Cultura Colli, ya que esta cultura se desarrolló en gran parte de la zona del valle bajo y medio durante el periodo Intermedio Tardío?

El sitio arqueológico Cerro Conde ubicado en la margen derecha del valle medio del río Chillón habría sido un asentamiento doméstico y administrativo. El sitio arqueológico cuenta con 2 sectores bien demarcados, el primero se encuentra sobre la ladera orientado de $\mathrm{S}$ a $\mathrm{N}$, que se dirige hacia la cumbre del cerro, donde se encuentra el otro sector. Las construcciones que se encuentran sobre la ladera del cerro están distribuidas de manera aglutinada, la concentración de recintos es en mayor cantidad a comparación de los recintos que se encuentran en la cumbre del cerro, estos recintos están construidos sobre terrazas y cuentan con pequeños pasajes entre 
recintos, el estado de conservación es malo a excepción de algunos recintos que se encuentran al lado NO de la ladera donde se pueden ver que los recintos cuentan con vanos orientado de $\mathrm{E}$ a $\mathrm{O}$.

Los recintos que se encuentran sobre la cumbre del cerro están sobre un terreno plano, donde hay recintos que están distribuidos en la parte central del terreno y otros a los bordes de la cumbre, estos recintos tienen plantas rectangulares y semicirculares, son más grandes a comparación con los recintos en la parte baja de la ladera. De esto se estaría planteando que el sector de la ladera estaría cumpliendo funciones domésticas y el sector de la cumbre, administrativa. Para demostrar que se trataba de un asentamiento doméstico y administrativo, se tendrá en cuenta indicadores como la presencia de cerámica doméstica, objetos de uso doméstico, arquitectura de centros de almacenes, arquitectura de centro de control, arquitecturas sobreelevadas.

Durante el Intermedio Tardío la sociedad Colli se desarrolló en la parte media y baja del río Chillón (Rostorowski, 1977), el sitio arqueológico Cerro Conde se encuentra dentro de estos límites de desarrollo de la cultura Colli, para demostrar que este sitio fue parte integrante de la cultura Colli se tendrá en cuenta indicadores como evidencia la cerámica del intermedio tardío asociado a la cultura colli y arquitectura tipo Colli. Si estos indicadores no concuerdan con los datos que se recuperan en campo se volverá a replantear.

\section{INVESTIGACIONES DEL PERIODO INTERMEDIO TARDÍO EN EL VALLE DEL CHILLÓN}

En el valle del río Chillón según lo que plantea Rostorowski, estaban asentadas diferentes etnias, de las cuales las más importantes fueron los Colli, los Guancayo, los Canta y los Atavillos (Rostorowsky, 1989). Señala que el Señorío de Colli era uno de las etnias más poderosas del valle bajo y que se pugnaban tierras con los Canta (Rostorowsky, 1989). Durante el Periodo Intermedio Tardío, para la cuenca alta del Chillón, Farfán (1992), menciona que este fue un escenario al igual que en los demás valles de los andes, un periodo de grandes cambios en el patrón de asentamiento y esto se debía al crecimiento poblacional, además de las grandes luchas de poder para el control del territorio y del agua.

En el año 1977, Tom Dillehay, investiga el sitio arqueológico Huancayo Alto, que se encuentra en el valle medio del río Chillón, hace un estudio de la funcionalidad del sitio y descubre que este sitio tuvo una ocupación desde el periodo Horizonte Temprano hasta el periodo colonial temprano.

Jorge Silva (1987) en su artículo "Patrones de asentamiento en el valle del Chillón”, cataloga que para el Intermedio tardío (1000-1440 d.C) en 3 clases a los sitios: 
a) Asentamientos sin muros perimétricos y sin protección natural, b) Asentamientos naturalmente protegidos y c) Asentamientos con muros perimétricos. En la cual hace un análisis de los diferentes asentamientos, con respecto al patrón arquitectónico y a los materiales constructivos; como los sitios de Trapiche (Pv46-824), Huatucay (Pv46674), Checta (Pv-954), Chocas (Pv46- 764), Cerro Conde (Pv46-671-673), entre otros.

Carlos Farfán Lobatón (2000) publica un artículo "La Ocupación Inca en Cantamarca, Canta", en donde hace una investigación minuciosa de este sitio, donde encuentra materiales relacionados a la dominación Inca en este sitio del intermedio tardío, además con las excavaciones que hizo nos detalla cómo fue la vivencia para los Cantamarca, en el valle alto del río Chillón. Posteriormente en el año 2014 junto a Cesar bautista Alderete y Antonio Raymondi Cárdenas, hacen un estudio de los patrones de asentamiento tardío en el valle del chillón, en donde analizan los diferentes sitios de este periodo, viendo su arquitectura y ubicación.

Posteriormente los estudios de Antonio Raymondi y Luisa Mejía Aranguren (2014) señalan que los grupos étnicos asentados en el valle bajo del río Chillón durante el Intermedio Tardío, estarían ordenados a través de un "modo de organización social agrupada" compuestos por montículos que se agrupaban alrededor de un Cerro Tutelar, esta área de influencia lo denominan como "Esferas Territoriales", en donde identifican 3 "Esferas Territoriales": Pro, Chuquitanta y Collique.

\section{ANTECEDENTES DE ESTUDIO DEL SITIO ARQUEOLÓGICO CERRO CONDE}

Jorge Silva (1987), define los asentamientos PV46-671, 672 y 673, en el Cerro Conde al norte de la ex Hacienda Huarangal, como un Asentamiento naturalmente protegido, ya que por su ubicación en la ladera del cerro Conde contaría con una visión panorámica del valle para su protección con otros grupos étnicos.

Silva en su tesis de Doctorado (1996) presentado en la Universidad de Michigan describe en una de sus prospecciones en el valle del Chillón, el sitio al que denomina a los sectores como PV-671/672/673, la cual hace una breve descripción de la arquitectura en superficie y el estado de conservación:

"PV46-671/673 sites were built with irregular cut stones, and even though no protecting walls were identified, their very locations make them protected places. PV46671 is on the southem slope of the Conde hill at about $50 \mathrm{~m}$ north of the ex- hacienda Huarangal. It is badly preserved but presents some evidence of rectangular rooms of 2 by $3 \mathrm{~m}$ set down on stone terraces. PV46-672 $(112,113)$ is north of 671 and covers an area of 1 hectare. Although they are also destroyed, rectangular and quadrangular rooms were placed on stone terraces. PV46-673 is a small group of Stone tenaces and 


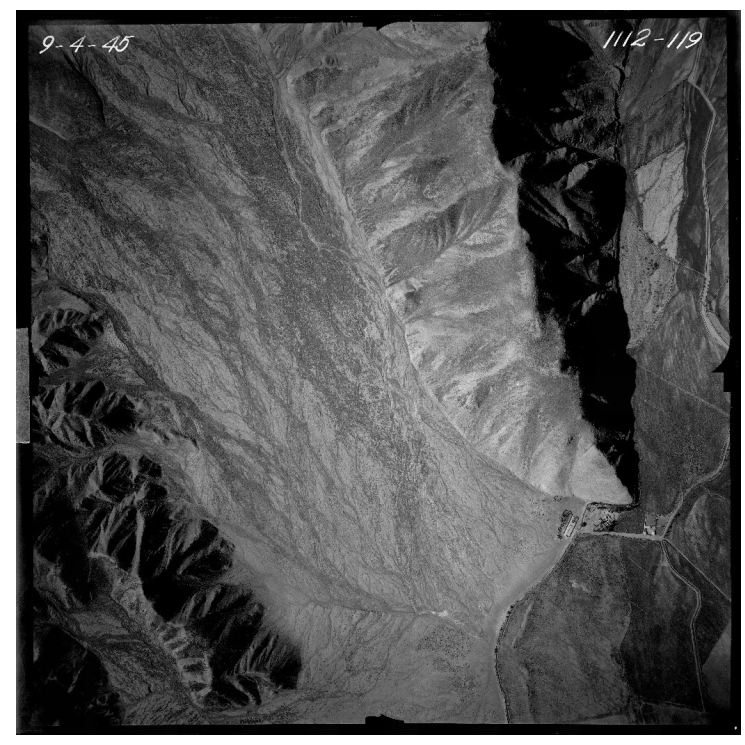

Figura $N^{\circ}$ 1. Fotografía aérea del sitio Arqueológico Cerro Conde, nótese al pie del cerro (lado Sur) la antigua Hacienda Huarangal. Fuente: Servicio Aerofotográfico Nacional, 1945.

walls $(2000 \mathrm{~m})$ are highly damaged. This last group of construction is at the eastem base of the Conde hill". (Silva, 1996).

Jorge Silva (1996) concluye que el sitio arqueológico Cerro Conde estaría cumpliendo funciones domesticas en la parte baja del cerro Conde, asociando a este la arquitectura aglutinada que se encuentra en esta parte del cerro y para la parte alta se estarían cumpliendo funciones ceremoniales. Además, sería un sitio con menor complejidad arquitectónica que Chocas Inferiores (PV46-707a-c, 708), y Chocas Superior (PV46-759a- b, 760, 764a-b, 765) y que este último estaría cumpliendo funciones administrativas.

Posteriormente Victoria Aranguren (2002) como parte de su tesis de Licenciatura, hace una mención del sitio en donde resalta que en la margen derecha del río Chillón, los sitios de Cerro Temblador, Cerro San Agustín, Cerro Conde y Macas A, las estructuras arquitectónicas están asociadas a alfarería del estilo Lima.

\section{RESULTADOS PRELIMINARES}

Durante las investigaciones se analizaron cuidadosamente cada estructura y se realizaron comparaciones a nivel arquitectónico con otros sitios arqueológicos con estructuras similares con la fortaleza de Collique, pudiendo identificarse algunas estructuras similares, como recintos cuadrangulares y terrazas. En cuanto al espacio en el que se emplaza y como se acentúa en el terreno agreste el sitio arqueológico Cerro Conde, se pudo identificar que Cerro Conde, cumple un patrón similar al de la Fortaleza de Collique ya que se emplaza en la ladera y cumbre de cerro. Cabe 
resaltar que durante los trabajos de campo en el sitio arqueológico Cerro Conde, no se encontraron restos de cerámica en los recintos ni en los espacios abiertos (plazas) no pudiéndose dar alguna cronología o filiación cultural Colli, dependiente de este análisis.

Cerro Conde es un sitio arqueológico que se encuentra emplazado en la ladera y cumbre del cerro, cuenta con diferentes espacios arquitectónicos con diferentes formas constructivas. Cerro Conde cumple el patrón general para los sitios asentados en el valle medio, que son emplazamiento en las partes elevadas del valle, teniendo a su alcance el manejo y control del río y de la zona productiva del valle. El sitio arqueológico Cerro Conde, cumple con algunas características en cuanto a nivel arquitectónico con otros sitios Colli y desplazamiento, como el caso de la Fortaleza de Collique (Cerro Macho). No pudiéndose hacer comparaciones en cuanto a fragmentos de cerámica por no encontrarse fragmentos de cerámica superficial en el Cerro Conde, no descartándose que por factores antrópicos y naturales se encuentre debajo de esta capa superficial.

Con los estudios que se han realizado por el momento se está tomando en cuenta el manejo arquitectónico y espacial del sitio Cerro Conde, además de la bibliografía consultada, para tratar de situarlo como un asentamiento del Intermedio Tardío asociado a la Cultura Colli, pero no teniendo una información en cuanto a otras variables como por ejemplo a la cerámica, no podemos determinarlo.

En cuanto a las funciones que se estarían realizando en el sitio arqueológico Cerro Conde, estas son diferenciadas, ya que se pudo identificar diferentes espacios arquitectónicos, que estarían siendo utilizados para diferentes tipos de actividades, como espacios para pernoctar en la parte baja del cerro, almacenamientos, espacios de control para la parte alta, pudiéndose corroborar que existían diferentes funciones en cuanto a la parte alta (cumbre del cerro) y parte baja (ladera del cerro). Otras de las características que se pudo identificar en cuanto a las distintas funciones que se estarían realizando en estos diferentes espacios es en la construcción diferenciada de las estructuras, en la parte baja del cerro se pudo identificar estructuras con planta rectangular y ovaladas en algunos casos, de menores dimensiones, sobre terrazas que se ajustan al terreno; además del sistema constructivo en cuanto que son muros de una sola hilera construidos de manera rustica ( sin argamasa). A diferencia de las estructuras de la parte alta del cerro que cuentan con estructuras con planta circulares, ovaladas, rectangulares con esquinas curvas, de mayores dimensiones, con una construcción mejor en cuanto a que son muros de doble hilera con rellenos de piedras. Cabe resaltar en cuanto al sostenimiento de las estructuras, que las terrazas están construidas con rellenos de piedras y sobre estos están construidos los recintos domésticos o de menor escala en la parte baja del sitio, dándole a estos recintos sostenimiento en cuanto al terreno en que se encuentran. En cuanto al sostenimiento que 
tendrían las estructuras en la parte alta del cerro (cumbre) que no cuentan con terrazas por estar en espacios planos, estos tendrían muros de doble hilera con rellenos de piedras para darle un mejor sostenimiento a las estructuras ya mencionadas. Con un estudio más detallado y excavaciones en el lugar se podría identificar el tipo de función específica que se estarían realizando en los diferentes espacios y estructuras arquitectónica, además de la filiación crono-cultural del sitio.

\section{REFERENCIAS BIBLIOGRÁFICAS}

Abanto, J. (2008). Lurigancho, un curacazgo Ychsma de La margen derecha del valle bajo del Rímac. Arqueología y Sociedad 19. Pp.159-177.

Anschuetz, K. F., Wilshusen, R. H., Scheick, R. H. (2001). An Archaology of Landscapes: Perspectives and Directions. Journal of Achaeological Research, vol 9(2), 152-197.

Aranguren Canales, V. (2002). Cerro Zapán: Una aldea Lima en el valle medio del Chillón. Universidad Nacional Federico Villarreal.

Canziani, J. (2003). Inicios del urbanismo en el Territorio Andino: Neolitización, primeros asentamientos aldeanos y arquitectura pública. URBES Revista de ciudad, urbanismo y paisaje, Volumen $\mathrm{N}^{\circ} 1$ Perú. Wiley Ludeña Urquizo.

Correa, I. (1992). Algunas consideraciones sobre la Fortaleza de Collique. Pachacamac, Revista del Museo de la Nación. 1(1), 139-142. Lima: Museo de la Nación.

Díaz, L. y Vallejo, F. (2004). Variaciones culturales en el valle de lima durante la ocupación incaica. Chungara, Revista de Antropología Chilena 36(2), 295-302.

De Sola-Morales I. (2000). Introducción a la Arquitectura: Conceptos Fundamentales. Barcelona. Carmen Rodríguez.

Dillehay, T. (1976). Competition and cooperation in a prehispanic multi-ethnic system in the Central Andes. Austin: Universidad de Texas.

Dillehay, T. (1987). Estrategias Políticas y económicas de las etnias locales del valle del Chillón durante el periodo prehispánico. Revista Andina 2, 407-456.

Eeckhout, P. (2008). Poder y jerarquías ychsmas en el valle de Lurín. Arqueología y Sociedad, 19, 223-240.

Farfán Lobatón, C. (2000). La Ocupación Inca en Cantamarca. En: Arqueología y Sociedad 3, 173 - 198. Museo de Antropología y Arqueología UNMSM.

Fundación Futuro Latinoamericano (FFLA). (2015). Proceso de conformación del Consejo de Recursos Hídricos de la Cuenca Interregional Chillón, Rímac y Lurín, Perú. Una experiencia de gobernanza. Quito-Ecuador. 
INRENA. (2003). Memoria Final. Estudios Integral de los Recursos Hídricos de la Cuenca del río Chillón. Hidrología Superficial. Lima- Perú.

Ludeña, Hugo. (1973). Investigaciones Arqueológicas en el sitio de Huacoy: Valle del Chillón. Tesis de Bachiller, Especialidad de Arqueología y Etnología, Universidad Nacional Mayor de San Marcos, Lima.

Lumbreras, L. G. (1974). The Peoples and Cultures of Ancient Peru. Washington: Smithsonian Institution Press. Traduit par Betty J. Meggers.

Mañana Borrazas, P. (2003). Arquitectura como percepción. Laboratorio de Arqueología, Instituto de Estudios Gallegos Padre Sarmiento. Arqueología de la Arquitectura 2, pp 117-183.

MINITERIO DEL AMBIENTE (MINAM). (2010). Informe final, Estudio Línea Base Ambiental de la Cuenca del Río Chillón. Ministerio del ambiente. Lima.

Morales, D. (1990). Proyecto de Investigación Arqueológica, Evaluación y Diagnóstico de la Fortaleza de Collique. Lima: Informe Final al Instituto Nacional de Cultura.

Murra, J. (1975). Formaciones económicas y políticas del mundo andino. Lima: Fondo Editorial del Instituto de Estudios Andinos.

Raymondi, A. y Mejía Aranguren, L. (2014). Ocupaciones prehispánicas tardías en el valle bajo del chillón: una aproximación desde la Huaca Pro. Arqueología y sociedad 28, 9-42

Raymondi, A. (2015). Territorio y Jerarquía tardía en el valle bajo del Chillón. LimaPerú. ArqueoWeb, 16. Pp. 146-175.

Rodríguez, A. (1999). Reconocimiento aero-arqueologico del complejo de geoglifos de la Quebrada de Torreblanca, valle del río Chillón Costa Central.

Rostworowski, M. (1977). Señoríos Indígenas de Lima y Canta. Lima: Instituto de Estudios Peruanos.

Rostworowski, M. (1978). Etnia y Sociedad. Costa Peruana Prehispánica. Lima: Instituto de Estudios Peruanos

Rostworowski, M. (1989). Historia del Tahuantinsuyo. Lima: Instituto de Estudios Peruanos.

Rowe, J. H., (1962). «Stage and Periods in archaeological interpretation». South wertern journal of anthropology 18, 40-54. California.

Silva, J. (1992.) Patrones de Asentamiento en el valle del Chillón. En: D. Bonavia (ed.) Estudios de Arqueología Peruana. Lima: FOMCIENCIAS.

Silva, J. (1996). Prehistoric Settlement Patterns in the Chillon River Valley, Peru. (Thesis Doctoral). Vol. I-II. University of Michigan. Doctoral dissetation. Departamento f Anthropology. Ann Arbor, Michigan. 
Silva, J. (1998). Una aproximación al periodo Formativo en el valle del Chillón. Boletín de Arqueología PUCP, 2. Pp. 251-268.

Silva, J. (2004). La Cultura Lima en el Valle del Río Chillón, Costa Central del Perú: Sitios y asentamientos pocos conocidos.

Stumer, L. (1953). Playa Grande Anticua eleganci en el Perú Pre- Tiahuanaco.

Stumer, L. (1954). "The Chillon Valley of Peru: excavation and reconnaissance-1952-1953 -Part 1». Archaeology, 7(3). Autum, Brattleboro Vermont.

Tácunan, Bonifacio. (2012). Los Colli una cultura poco conocida del valle del Chillón. Collique. Historia de un pueblo solidario. Editorial de la Universidad Católica Sedes Sapientiae. Págs.1-23.

van Dalen Luna, P. (2012). Arqueología Tardía del Valle Chancay-Huaral: Identificando la nación Chancay. Investigaciones Sociales 16(28), 271-283. UNMSM-IIHS. LIMA, PERÚ.

Vera Hernández, L. (2002). Análisis de aforo de la estación hidrométrica Obrajillo- Periodo 200-2001. Informe Profesional para optar el Título Profesional de Ingeniero de Fluidos. E.A.P. Ingeniería Mecánica de Fluidos. UNMSM, Lima.

Villar Córdova, P. (1935). Las culturas prehispánicas del departamento de Lima. Atusparia, Lima.

\section{SOBRE EL AUTOR}

\section{ANTHONY ULISES VILLALTA TELLO}

Bachiller en Arqueología por la Universidad Nacional Mayor de San Marcos. Es miembro Adherente del grupo de Investigación: Arqueología Andina y Gestión de Recursos Culturales y Naturales (ARQANBIO). Tiene experiencia como arqueólogo de campo y gabinete. Ha realizado trabajos de campo en diferentes regiones del país. Actualmente viene desempeñando trabajos de investigación como arqueólogo de campo en la provincia de Huaral. 